\title{
Topoisomerase I copy number alterations as biomarker for irinotecan efficacy in metastatic colorectal cancer
}

\author{
Jesper Andreas Palshof ${ }^{*}$, Estrid Vilma Solyom Høgdall², Tim Svenstrup Poulsen², Dorte Linnemann², \\ Benny Vittrup Jensen ${ }^{1}$, Per Pfeiffer ${ }^{3}$, Line Schmidt Tarpgaard ${ }^{3}$, Nils Brünner ${ }^{4}$, Jan Stenvang ${ }^{4}$, Mette Yilmaz ${ }^{5}$ \\ and Dorte Lisbet Nielsen'
}

\begin{abstract}
Background: No biomarker exists to guide the optimal choice of chemotherapy for patients with metastatic colorectal cancer. We examined the copy numbers (CN) of topoisomerase I (TOP1) as well as the ratios of TOP1/ CEN-20 and TOP1/CEN-2 as biomarkers for irinotecan efficacy in patients with metastatic colorectal cancer.

Methods: From a national cohort, we identified 163 patients treated every third week with irinotecan $350 \mathrm{mg} / \mathrm{m}^{2}$ as second-line therapy. Among these 108 were eligible for analyses and thus entered the study. Primary tumors samples were collected and tissue microarray (TMA) blocks were produced. FISH analysis was performed using two probe-mixes: TOP1/CEN-20 and TOP1/CEN-2. Only samples harboring all three signals (TOP1, CEN-20 and CEN-2) using FISH were included in the analyses.

Results: In the TOP1/CEN-20 probe-mix the median TOP1- and CEN-20 CN were 4.46 (range: 1.5-9.5) and 2.00 (range: $0.55-4.55$ ), respectively. The median TOP1- and CEN-2 CN in the TOP1/CEN-2 probe-mix, were 4.57 (range: 1. 82-10.43) and 1.98 (range: 1.22-6.14), respectively. The median TOP1/CEN-20 ratio and TOP1/CEN-2 ratio were 1.25 (range: 0.92-2.90) and 2.05 (range: 1.00-6.00), respectively. None of the markers TOP1 CN, TOP1/CEN-20-ratio or TOP1/CEN-2-ratio were associated with progression free survival, overall survival or baseline characteristics. Yet, we observed a borderline association for a stepwise increase of the TOP1 CN in relation to objective response as hazard ratio were 1.35 (95\% Cl 0.96-1.90; $p=0.081)$.

Conclusions: We verified a borderline significant association between increasing TOP1 CN and objective response as previously reported. Applying the probes representing CEN-20 and CEN-2, in order to investigate the ratios of TOP1/CEN-20 and TOP1/CEN-2 provided no further information in search of a biomarker driven patient stratification. Other biomarkers to be paired with TOP1 CN are therefore highly warranted.
\end{abstract}

Keywords: Biomarker, Colorectal cancer, FISH, Gene copy number, Irinotecan, Topoisomerase I

\section{Background}

Colorectal cancer (CRC) is the third most common cancer and the fourth most common cause of cancer death worldwide [1]. Almost $50 \%$ of patients diagnosed with CRC will develop metastatic disease [2]. Standard of care for patients with non-resectable metastatic colorectal cancer (mCRC) is combination chemotherapy with 5-fluorouracil (5-FU)/

\footnotetext{
* Correspondence: jesper.andreas.palshof@regionh.dk

'Department of Oncology, Herlev Hospital, University of Copenhagen, Herlev Ringvej 75, DK-2730 Herlev, Denmark

Full list of author information is available at the end of the article
}

leucovorin (LV)/oxaliplatin (FOLFOX) or 5-FU/LV/irinotecan (FOLFIRI) with or without a targeted agent [3]. In firstline therapy, FOLFIRI and FOLFOX are considered equally effective [4].

Predictive biomarkers for the efficacy of 5-FU, irinotecan and oxaliplatin have been suggested but none, so far, have been implemented in the clinical setting [5]. However, a significant fraction of the patients does not benefit from the treatment but may experience serious side effects only. The topoisomerase 1 (Top1) protein is an essential nuclear enzyme for vital cellular processes such 
as DNA replication, transcription, translation, recombination and repair. The primary function of Top1 is to unwind and uncoil the supercoiled DNA double helix by transiently cleaving one of the two strands and thereby allowing its rotation over the other strand [6]. This intermediate cleavage state is termed the Top1 cleavage complex (Top1cc). Top1 is the target of irinotecan (CPT-11), a camptothecin derivative, which is metabolized to the active metabolite $\mathrm{SN}-38$ which binds to and stabilizes the Top1cc, whereby the rapidly moving DNA replication and transcription complexes collide with this trapped Top1ccs. The main cytotoxicity induced by irinotecan is caused by DNA double-strand breaks during DNA replication and the presence of Top1 is thus a prerequisite for this cytotoxic effect $[7,8]$.

The plausible link between tumor tissue levels of Top1 and effect of Top1 inhibitors in cancer treatment [9] has been investigated by different methods. In vitro studies with colon cancer cell lines have demonstrated a significant correlation between the topoisomerase I gene (TOP1) copy numbers $(\mathrm{CN})$ or Top1 protein expression and the sensitivity to $\mathrm{SN}-38[9,10]$. A prospective clinical trial (FOCUS) investigated the association between Top1 protein expression and benefit from FOLFOX and FOLFIRI in patients with mCRC [11]. A significant association between Top1 protein immunoreactivity and clinical benefit from FOLFIRI or FOLFOX was found. However, conflicting results have been reported since the findings could not be validated in a subsequent study from the same group (FOCUS3) [12]. Furthermore, the results from another large prospective trial (CAIRO) showed no correlation of Top1 immunoreactivity and response to irinotecan in patients with mCRC [13]. An explanation to these diverse results was recently provided by (Maughan et al.) who showed that the antibody used for IHC in the above mentioned studies did not result in reproducible staining patterns [12]. We have recently introduced another approach for Top1 quantitation in cancer cells. Instead of immunohistochemistry IHC to quantitate protein expression we used fluorescence in situ hybridization (FISH) to assess TOP1 gene copy number $(\mathrm{CN})$ status as a proxy for the overall Top1 protein levels. We have previously identified a significant correlation between the TOP1 gene CN, TOP1 mRNA expression and Top1 protein levels using data generated from in vitro studies on CRC cell lines [14].

The (TOP1) gene is located on chromosome 20 at $20 \mathrm{q} 12$ and this region frequently undergoes $\mathrm{CN}$ alterations in various cancers [14-17]. In CRC, the TOP1 aberration has been reported by applying a TOP1/CEN20 fluorescence in situ hybridization (FISH) probe-mix. The TOP1 CN gain in CRC has been reported to be in the range of $53-84 \%$, whereas TOP $1 / \mathrm{CEN}-20$ ratios $\geq 1.5$ or $\geq 2.0$ were in the range of $30-40 \%$ and $10-20 \%$, respectively $[14,18,19]$. Current data suggests that TOP1 CN increases occur predominately in conjunction with the rest of $20 \mathrm{q}[14,16,17,20]$ and the CEN-20 region $[14,18]$. Therefore the usage of the TOP1/CEN-20 ratio may underestimate the genuine TOP1 amplifications. Chromosome 2 (CEN-2) has been found to be the least affected by independent numeric aberrations in the genome, and has therefore been combined with TOP1 in a TOP1/ CEN-2 probe-mix to distinguish between TOP1 gene gain and genuine TOP1 amplifications [21]. These two different types of $\mathrm{CN}$ alterations have been demonstrated to have differential prognostic effects in stage III CRC patients [21]. In a metastatic setting a borderline significant association $(p=0.07)$ between an increase in TOP1 CN and objective response to second-line treatment with irinotecan monotherapy has been reported [19].

Therefore, we applied both a TOP1/CEN-20 and a TOP1/CEN-2 FISH probe-mix to 108 tumors from $\mathrm{mCRC}$ with the aim to investigate TOP1 $\mathrm{CN}$ and the ratios of both TOP1/CEN-20 and TOP1/CEN-2 as biomarkers for irinotecan efficacy.

\section{Methods}

The patients included in this explorative study were extracted from a national cohort of 498 patients with mCRC who all received irinotecan in combination with the epidermal growth factor receptor inhibitor, cetuximab as third-line treatment from 1st of January 2005 to 1st of August 2008 at the Departments of Oncology at Herlev, Odense, and Aalborg Hospitals in Denmark. The inclusion period for the national cohort was specifically selected because it preceded the introduction of KRAS testing. Therefore, mutational status for KRAS, NRAS and BRAF is unknown in this cohort. From this cohort we identified 163 consecutive patients who were treated every third week with irinotecan $350 \mathrm{mg} / \mathrm{m}^{2}$ as second-line therapy. Disease evaluation was performed during treatment using computed tomography (CT) scans of the thorax and abdomen every 9-12 weeks to evaluate response according to the RECIST 1.0 criteria [22]. Data for objective response, progression-free survival (PFS) and overall survival (OS) were extracted from the database. PFS was defined as time from start of treatment to progression or death from any cause. OS was defined as time from start of treatment to death from any cause. Last follow-up on survival was done in October 2014. The study was approved by the Research Ethics Committee of Copenhagen (H-KA-20060094). Reporting of the results was prepared according to the REMARK criteria [23].

\section{Tumor material}

Only primary tumor samples were used. Formalin-fixed paraffin-embedded samples from either resections or core needle biopsies were collected. The presence of 
tumor cells in the samples was confirmed by a pathologic review performed by JP and an experienced gastrointestinal pathologist (DL). Tissue microarray (TMA) blocks were produced; each containing tumor material from 18 patients with two $1 \mathrm{~mm}$ tissue cores per patient sample. Standard procedures were used for preparation of the TMA blocks. The TMA blocks were cut in $2-\mu \mathrm{m}$ sections and stored at $5{ }^{\circ} \mathrm{C}$ until hybridization.

\section{Fluorescence in situ hybridization (FISH)}

The probes for TOP1, CEN-20 and CEN-2 were developed and produced by the Department of Pathology, Herlev University Hospital. All probes were sequenced to confirm that all base pairs exactly matched the TOP1 gene and the centromeres CEN-20 and CEN-2. Two probe-mixes: $T O P 1 / \mathrm{CEN}-20$ and TOP1/CEN-2 were produced. The probes were labelled with Texas red (TOP1) and fluorescein isothiocyanate (FITC), green for CEN-20 and CEN-2. Only samples harboring all three signals (TOP1, CEN-20 and CEN-2) using FISH were included in the analyses. Since the TOP1 probe was present in both probe-mixes, it was counted twice - independently.

Two slides from each TMA block were deparaffinized, rehydrated, boiled in pre-treatment buffer for $10 \mathrm{~min}$ and cooled in the buffer for $15 \mathrm{~min}$ at room temperature followed by $2 \times 3 \mathrm{~min}$ in wash buffer (1:20) (K5799 Dako). RTU-pepsin was added for 2 min at $37^{\circ} \mathrm{C}$ and removed in wash buffer for $2 \times 3 \mathrm{~min}$. Following ethanol $(70 \% \rightarrow 96 \% \rightarrow 99 \%)$ dehydration and $15 \mathrm{~min}$. air-dry,
$10 \mu \mathrm{L}$ of TOP1/CEN-20 probe was applied to the center of one of the two slides and $10 \mu \mathrm{L}$ of TOP1/CEN-2 probe-mix was applied to the other slide. Non-specific binding of probe was removed by stringency wash (1:20) at $65{ }^{\circ} \mathrm{C}$ for $10 \mathrm{~min}$ (K5731 - Dako). A fluorescence microscope (Olympus BX61) with DAPI, FITC, Texas Red and dual FITC/Texas Red filter was used for visualization of the signals. Signal counting was performed by JP, blinded to all patient data. In case of ambiguity, a senior pathologist (DL) was consulted. A minimum of sixty TOP1 signals in total, 30 from each of the two cores, were counted in non-overlapping cancer nuclei with well-defined morphology and distinct fluorescent signals. If the fluorescent intensity was weak or insufficient tumor tissue was present, a new section was cut. If signals continued to be too weak for clear interpretation, the sample was excluded from the analyses.

\section{Cutoffs and Definitions}

A cutoff of 2 for the ratios of TOP1/CEN-20 and TOP1/ CEN-2 were used in this study.

Tumors were classified as "amplified" when (TOP1/ $\mathrm{CEN}-20$ ratio $\geq 2$ and TOP1/CEN-2 ratio $\geq 2$ ) and as "polysomies" when (TOP1/CEN-20 ratio $\leq 2$ and TOP1/ CEN-2 ratio $\geq 2$ ).

\section{Statistics}

To examine the association between TOP1 $\mathrm{CN}$ from the two probe-mixes, a Reliability Analysis with an Intraclass

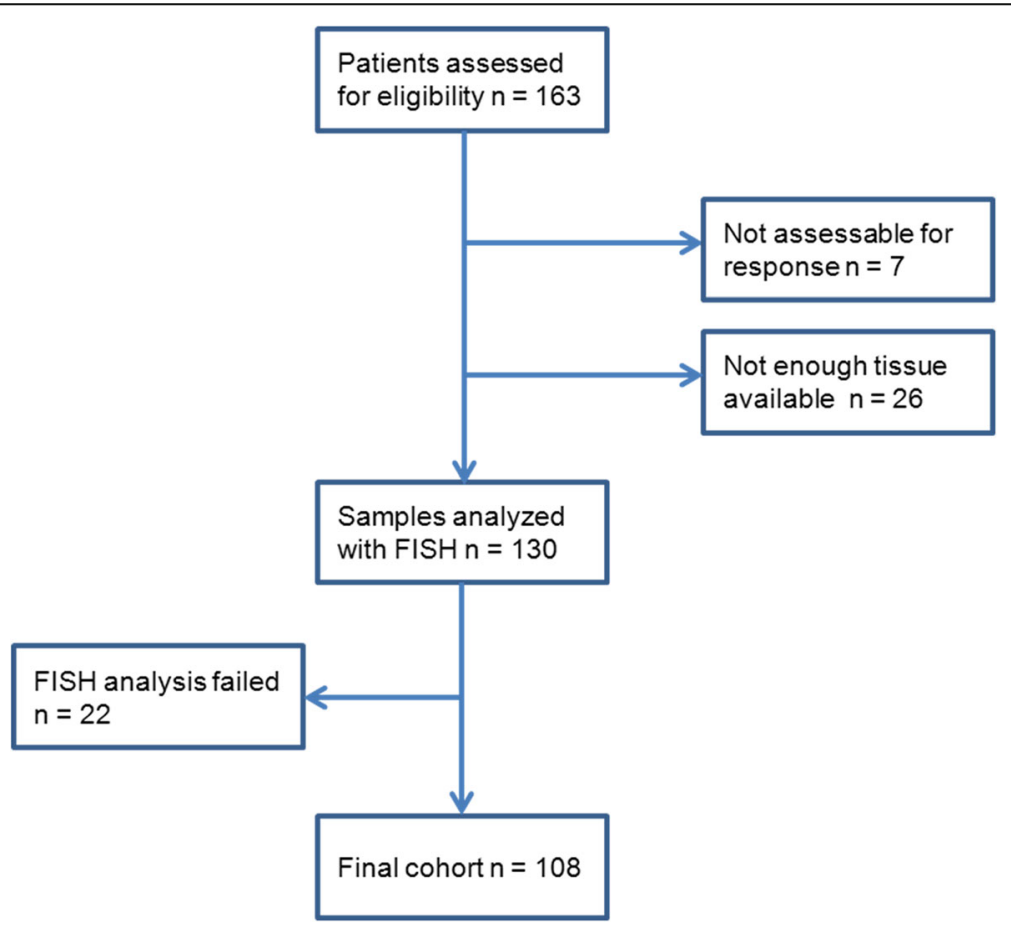

Fig. 1 CONSORT diagram showing the flow of patients and samples 


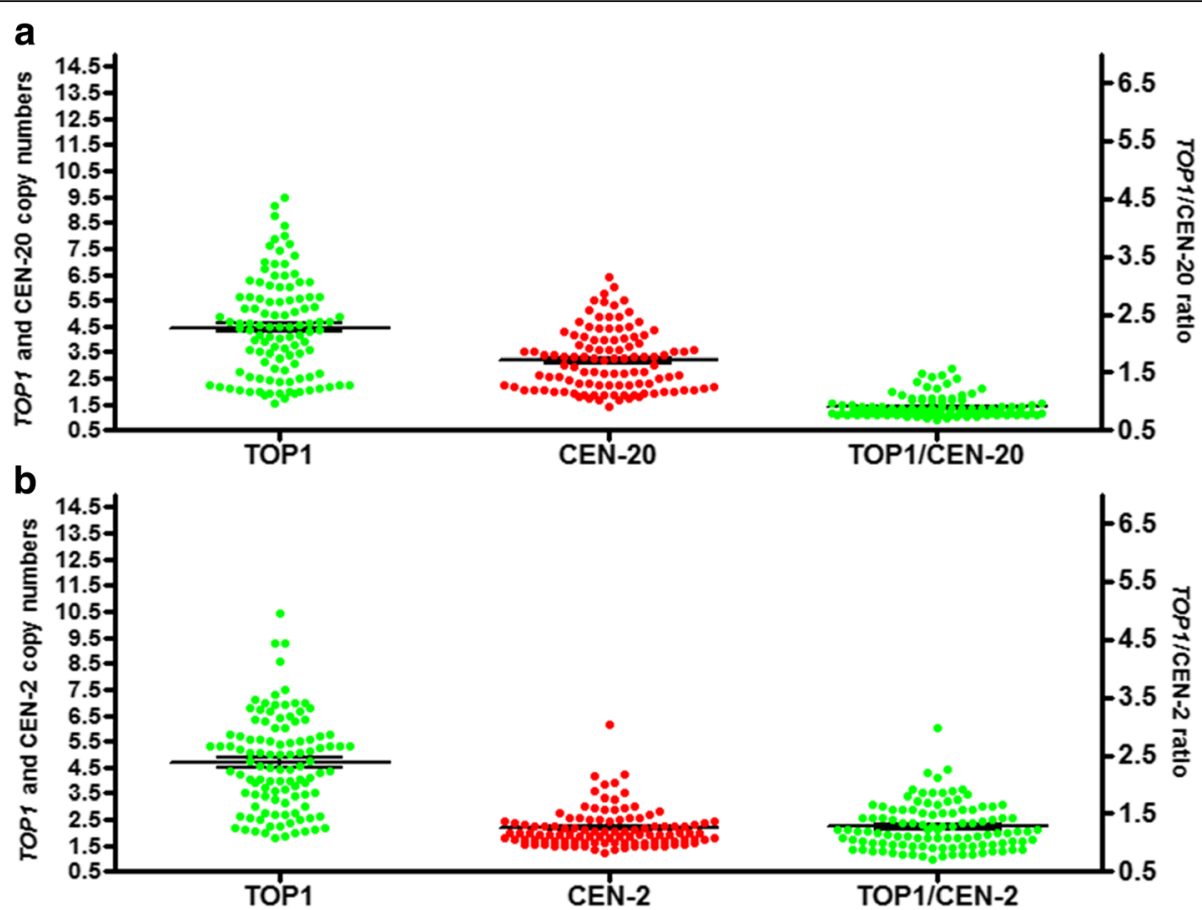

Fig. 2 Distribution of TOP1, CEN-20 and CEN-2 copy numbers and of TOP1/CEN-20 and TOP1/CEN-2 ratios. Distribution in colorectal cancer samples determined by FISH. a Showing the distribution of the copy numbers for probe-mix TOP1/CEN-20. b Showing the distribution of the copy numbers for probe-mix TOP1/CEN-2

correlation was performed. Pearson's chi-squared test was used to test for associations between baseline characteristics and TOP1 CN and TOP1-/CEN-20- and CEN-2 ratios. The baseline characteristics were: gender, age, WHO performance status (PS), location of primary tumor, resection of primary tumor, number of metastatic sites, prior chemo- and radiotherapy, and presence of lung or liver metastases. The TOP $1 \mathrm{CN}$ per cell was divided by the median value into two groups. TOP1/CEN-20- and CEN-2 ratios were divided into $\geq 2$ and $<2$. $p$-values and $95 \%$ confidence intervals $(\mathrm{CI})$ are reported.

The final analyses of survival data were performed in October 2014. Cox proportional hazards (CPH) regression models were used to test for association between time-toevent endpoints and both a dichotomized value and a continuous value of TOP1 signal count, TOP1/CEN-20 ratio, and TOP1/CEN-2 ratio, with adjustment for baseline characteristics. We also used $\mathrm{CPH}$ models to test "amplified" (TOP1/CEN-20 ratio $\geq 2$ and TOP1/CEN-2 ratio $\geq 2$ ) vs "non- amplified" tumors and "polysomies" (TOP1/ CEN-20 ratio $\leq 2$ and TOP1/CEN-2 ratio $\geq 2$ ) vs "non-polysomies". Wald chi-squared $p$-values hazard ratio (HR) estimates with estimated 95\% CI interval were reported.

The association between TOP1 CN, TOP1/CEN-20 ratio, and TOP1/CEN-2 ratio and objective response rate was tested using logistic regression. Responders were defined as patients with complete (CR) or partial response (PR) according to RECIST 1.0 criteria. We also performed the same analysis using clinical benefit rate (CBR). Clinical benefit was defined as CR, PR or stable disease (SD) $\geq 6$ months. For these tests, Wald chi-squared $p$-values and odds ratio (OR) estimates with 95\% CI were reported.

\section{Results}

In the database of our national cohort, we searched for patients having received irinotecan monotherapy as secondline therapy. We identified 163 patients. Of the 163, 26 (16\%) patients were excluded as they did not have enough tissue available for analyses, in $22(13 \%)$ we failed to produce countable FISH signals from both probe-mix (TOP1/ CEN-20 and TOP1/CEN-2) after two attempts and 7 patients were not assessable for response due to either concurrent surgery or radiotherapy. Thus, 108 patients were eligible for analyses in the study (Fig. 1).

Table 1 Distribution of TOP1 CN and ratios for the 108 patients

\begin{tabular}{llll}
\hline & $\begin{array}{l}\text { TOP1 CN } \\
\text { No (\%) }\end{array}$ & $\begin{array}{l}\text { TOP1/CEN-2 } \\
\text { ratio No (\%) }\end{array}$ & $\begin{array}{l}\text { TOP1/CEN-20 } \\
\text { ratio No (\%) }\end{array}$ \\
\hline$<2$ & $7(6)$ & $50(46)$ & $98(91)$ \\
$2.0-2.99$ & $23(21)$ & $36(33)$ & $10(9)$ \\
$3.0-3.99$ & $14(13)$ & $18(17)$ & - \\
$4.0-4.99$ & $25(23)$ & $3(3)$ & - \\
$5.0-5.99$ & $15(14)$ & - & - \\
$6.0-6.99$ & $13(12)$ & $1(1)$ & - \\
$>7$ & $11(10)$ & - & - \\
\hline
\end{tabular}




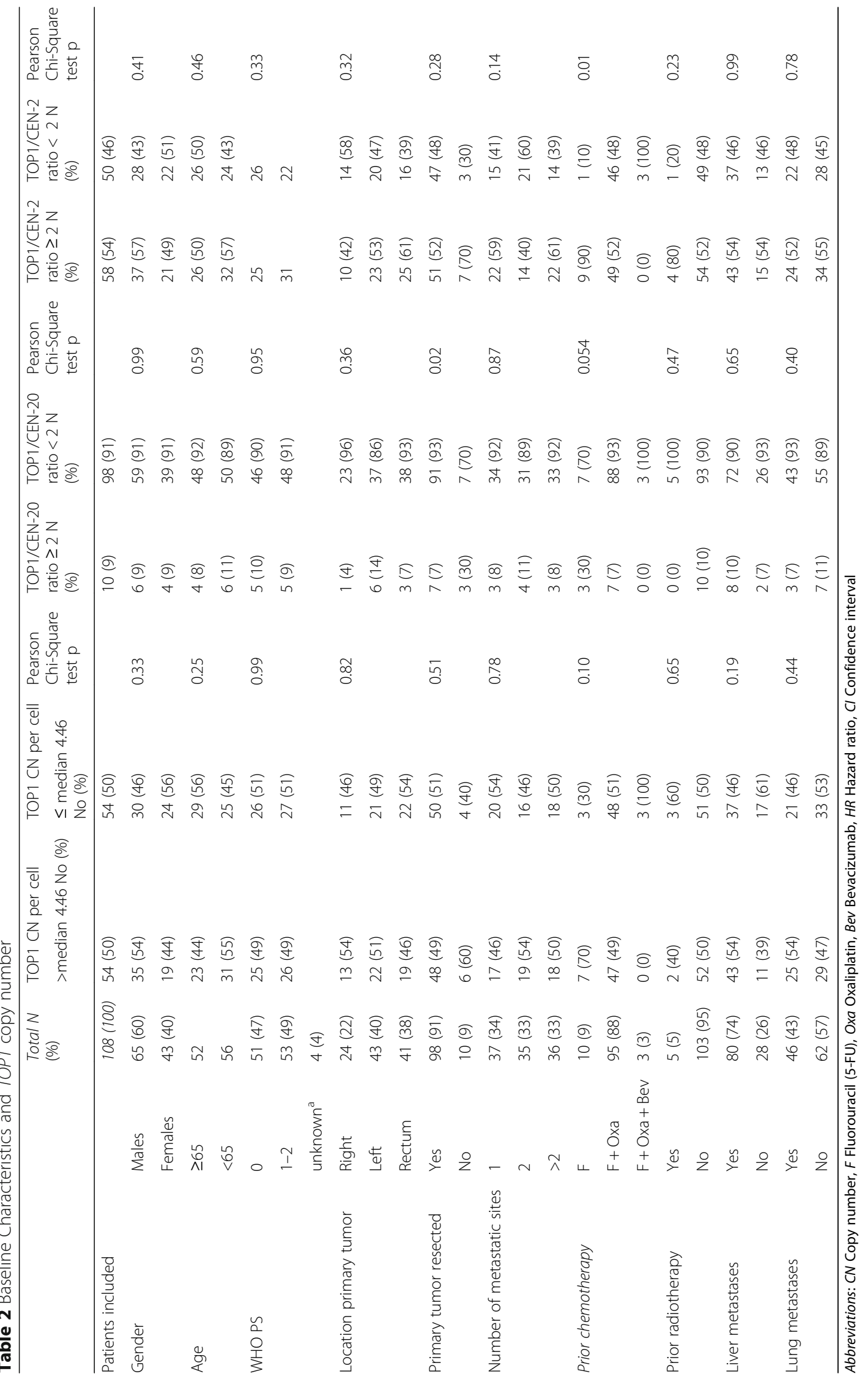


TOP1 CN was counted twice due to the use of two probe-mixes. When comparing the results of TOP1 CN from the two probe-mixes, the Single Measures Intraclass correlation was $\mathrm{r}=0.74$ (CI 0.64-0.82; $p<0.001$ ). The Spearman correlation between TOP1 and CEN-2 was: $\mathrm{r}=0.44 \quad(p<0.001)$, between TOP1 and CEN-20: $\mathrm{r}=0.82(p<0.001)$ and between CEN-2 and CEN-20: $\mathrm{r}=0.41 \quad(p<0.001)$.

For the TOP1/CEN-20 probe-mix, the median TOP1- and CEN-20 CN were 4.46 (range: 1.5-9.5) and 2.00 (range: $0.55-4.55$ ), respectively. The median TOP1 - and CEN-2 CN in the TOP1/CEN-2 probemix, were 4.57 (range: 1.82-10.43) and 1.98 (range: 1.22-6.14), respectively. The median TOP1/CEN-20 ratio and TOP1/CEN-2 ratio were 1.25 (range: 0.922.90 ) and 2.05 (range: 1.00-6.00), respectively (Fig. 2). The distribution of TOP1 CN and ratios for the 108 patients are shown in Table 1 . We used the median TOP1 CN (probe-mix TOP1/CEN-20) to test the association with baseline characteristics (Table 2). Significant associations between prior chemotherapy and TOP $1 / \mathrm{CEN}-2$ ratio and between liver metastases and TOP1/CEN-20 ratio were found. However, these results were not significant after correction for multiple testing (data not shown).

Ten patients (9\%) had PR, 46 (43\%) had SD, and 51 (47\%) had PD as best response. The distribution of TOP1 CN for patients having PR, SD and PD is illustrated in Fig. 3. The OR estimates for a stepwise increase of the TOP1 CN and TOP1/CEN-20 and TOP1/CEN-2 ratios in relation to objective response were 1.35 (CI $0.96-1.90 ; p=0.081$ ), 1.99 (CI 0.51-7.75; $p=0.32$ ), and 1.34 (CI $0.69-2.64 ; p=0.40$ ), respectively. No significant association was found between $\mathrm{CBR}$ and a stepwise increase of the TOP1 CN, TOP1/CEN-20- or TOP1/ CEN-2-ratio as the OR estimates were 1.01 (CI 0.81$1.25 ; p=0.94), 0.72$ (CI $0.24-2.14 ; p=0.56$ ), and 0.88 (CI $0.54-1.42 ; p=0.60$ ), respectively.

The median PFS and OS were 3.8 months (range: 1.313.1 ) and 16.4 months (range: 4.6-91.6), respectively. None of the biomarkers TOP1 CN, TOP1/CEN-20-ratio or TOP1/CEN-2-ratio when tested as continuous variables, were associated with PFS as HRs were 0.99 (CI 0.90-1.10; $p=0.88$ ), 0.99 (CI 0.56-1.74; $p=0.97$ ), and 1.10 (CI 0.85$1.35 ; p=0.58)$, respectively. For OS, HRs were 0.98 (CI $0.89-1.08 ; p=0.72$ ), 1.02 (CI $0.61-1.70 ; p=0.95$ ), and 1.00 (CI 0.79-1.27; $p=0.98$ ) for TOP1 CN, TOP1/CEN-20-ratio and TOP1/CEN-2-ratio, respectively (Table 3).

We did not find any associations between the dichotomized version of TOP1 $\mathrm{CN}$ and PFS and OS as HRs were 0.95 (CI $0.65-1.40 ; p=0.79$ ) and 0.92 (CI $0.62-$ $1.35 ; p=0.66)$, respectively. We also tested PFS as a function of TOP1 $\mathrm{CN}$ divided into tertiles and found no significant association, log-rank $p=0.66$ (Fig. 4).

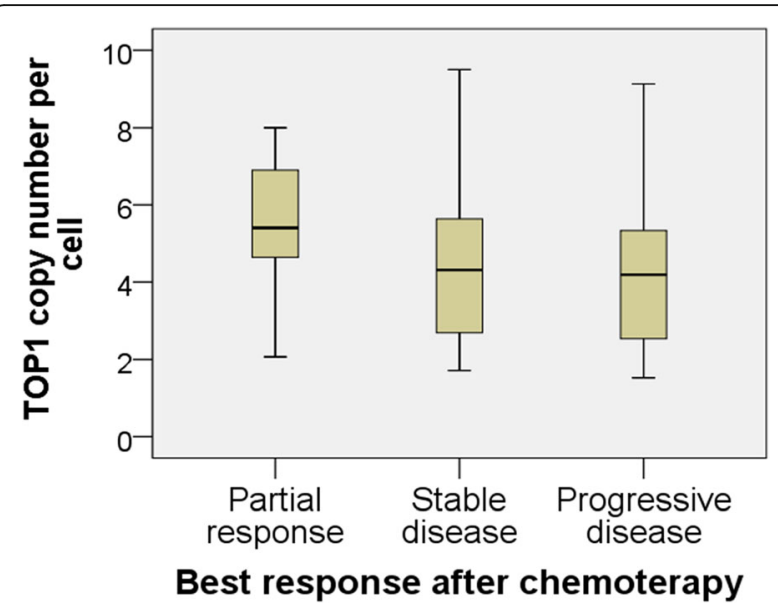

Fig. 3 Boxplot. Distribution of TOP1 copy number as a function of best response to chemotherapy. The top and bottom of the box represents the upper and lower quartiles and the black line in the box the median. The whiskers represent the maximum and minimum values, excluding outliers

According to the definitions used, 9\% had an amplified tumor (TOP1/CEN-20 ratio $\geq 2$ and TOP $1 / \mathrm{CEN}-2$ ratio $\geq 2$ ), $44 \%$ had a tumor harboring a q-20 polysomy $(T O P 1 / C E N-20$ ratio $\leq 2$ and TOP1/CEN-2 ratio $\geq 2$ ), and $46 \%$ had a tumor that was neither amplified nor a polysomy. Patients with tumors classified as harboring a TOP1 amplification did not have improved PFS: HR 1.71 (CI $0.88-3.32$; $p=0.12$ ) or OS: HR 1.32 (CI 0.69 $2.54 ; p=0.41)$. Neither did patients with tumors classified as harboring q-20 TOP1 polysomy: PFS HR 0.94 (CI 0.64-1.38; $p=0.75$ ) and OS HR 0.71 (CI 0.48-1.05; $p=0.08)$. There were no significant associations between TOP1 amplification or q-20 TOP1 polysomy and objective response.

No multivariate analysis was performed since the variables tested were not significant in the univariate analyses.

\section{Discussion}

This study is the first to report the CN of TOP1 and the ratios of TOP1/CEN-20 and TOP1/CEN-2 in CRC patients and their relation to objective response to irinotecan in a metastatic setting. We added a probe for CEN-2 to discriminate between an increase in TOP1 $\mathrm{CN}$ due to mechanisms localized to chromosome 20 and an increase caused by increased ploidy level. We chose chromosome 2 because this chromosome has been reported to undergo fewer alterations when compared to other chromosomes in cancer specimens [24]. In accordance with this, a recent study investigating TOP1 $\mathrm{CN}$ aberrations, considered $\mathrm{CEN}-20 \mathrm{CN}$ as an inappropriate 
Table 3 Univariate survival analyses

\begin{tabular}{|c|c|c|c|c|c|c|c|}
\hline & & Progr & ee survival & & Overa & & \\
\hline & & $H R$ & $95 \% \mathrm{Cl}$ & $p$ & $\mathrm{HR}$ & $95 \% \mathrm{Cl}$ & $p$ \\
\hline Gender & Females & (ref) & & & (ref) & & 0.03 \\
\hline & Males & 0.65 & $0.43-0.96$ & 0.03 & 0.64 & $0.43-0.94$ & \\
\hline Age & Per 1 year increase & 0.99 & $0.97-1.02$ & 0.63 & 0.98 & $0.96-1.01$ & 0.16 \\
\hline WHO PS & 0 & 0.84 & $0.57-1.24$ & 0.38 & 0.59 & $0.39-0.88$ & 0.009 \\
\hline & $1-2$ & (ref) & & & (ref) & & \\
\hline & unknown $^{a}$ & & & & & & \\
\hline Location primary tumor & Right & 0.74 & $0.45-1.23$ & 0.25 & 1.21 & $0.72-2.02$ & 0.48 \\
\hline & Left & 0.87 & $0.57-1.35$ & 0.54 & 1.14 & $0.74-1.76$ & 0.55 \\
\hline & Rectum & (ref) & & & (ref) & & \\
\hline Primary tumor resected & Yes & 0.78 & $0.41-1.51$ & 0.46 & 0.78 & $0.40-1.50$ & 0.49 \\
\hline & No & (ref) & & & (ref) & & \\
\hline Number of metastatic sites & 1 & (ref) & & & (ref) & & \\
\hline & 2 & 1.05 & $0.66-1.67$ & 0.84 & 1.31 & $0.82-2.10$ & 0.27 \\
\hline & $>2$ & 1.23 & $0.77-1.96$ & 0.40 & 1.51 & $0.95-2.40$ & 0.09 \\
\hline Prior chemotherapy & $\mathrm{F}$ & (ref) & & & (ref) & & \\
\hline & $\mathrm{F}+\mathrm{Oxa}$ & 0.99 & $0.52-1.92$ & 0.99 & 2.50 & $1.25-5.00$ & 0.009 \\
\hline & $\mathrm{F}+\mathrm{Oxa}+\mathrm{Bev}$ & 1.91 & $0.52-6.96$ & 0.33 & 3.53 & $0.94-13.2$ & 0.06 \\
\hline Prior radiotherapy & Yes & 0.71 & $0.29-1.75$ & 0.46 & 0.85 & $0.35-2.10$ & 0.73 \\
\hline & No & (ref) & & & (ref) & & \\
\hline Liver metastases & Yes & 0.88 & $0.57-1.37$ & 0.58 & 1.18 & $0.76-1.82$ & 0.64 \\
\hline & No & (ref) & & & (ref) & & \\
\hline Lung metastases & Yes & 1.09 & $0.73-1.61$ & 0.67 & 1.50 & $1.01-2.21$ & 0.044 \\
\hline & No & (ref) & & & (ref) & & \\
\hline TOP1 CN (CEN-20) & Per 1 unit increase & 0.99 & $0.90-1.10$ & 0.88 & 0.98 & $0.89-1.08$ & 0.72 \\
\hline TOP1 CN (CEN-20) & >median (4.46) & 0.95 & $0.65-1.40$ & 0.79 & 0.92 & $0.62-1.35$ & 0.66 \\
\hline & $\leq$ median $(4,46)$ & (ref) & & & (ref) & & \\
\hline TOP1/CEN-20 ratio & Per 1 unit increase & 0.99 & $0.56-1.74$ & 0.97 & 1.02 & $0.61-1.70$ & 0.95 \\
\hline TOP1/CEN-2 ratio & Per 1 unit increase & 1.10 & $0.85-1.35$ & 0.58 & 1.00 & $0.79-1.27$ & 0.98 \\
\hline TOP1/CEN-20 ratio & $<1.5$ (ref) & & & & & & \\
\hline & $\geq 1.5$ & 0.73 & $0.47-1.13$ & 0.16 & 0.75 & $0.48-1.17$ & 0.21 \\
\hline
\end{tabular}

Abbreviations: F Fluorouracil (5-FU), Oxa Oxaliplatin, Bev Bevacizumab, HR Hazard ratio, Cl Confidence interval

a Unknowns were not included in the shown proportions or test for distribution differences

marker for cellular ploidy based on the frequent gain of chromosome 20 or 20q [21].

TOP1 cut-off values were chosen based on the median value. This decision was partly based on the results from a similar study [25] and partly to obtain two equally sized groups. In our study, we found a higher median gene $\mathrm{CN}$ for TOP1 (4.46) than reported in the study by Nygaard et al. (3.6) [25]. A plausible explanation for this discrepancy could be the difference in number of cells counted. In our study we counted a minimum of 60 signals of TOP1 $\mathrm{CN}$ for each patient giving a median of 14.5 and a range of $8-40$ counted nuclei. This is less than the 60 nuclei counted by Nygaard et al. One could argue that since we selected the nuclei with the best quality signals for counting we also tended to select the nuclei with the highest CNs. Despite the difference in methods, our study showed the same results as Nygaard et al. concerning the association with objective response, PFS, and OS.

Previously, a positive correlation between the TOP1 $\mathrm{CN}$ and in vitro sensitivity to $\mathrm{SN}-38$ was reported in CRC cell lines and this was also demonstrated for the TOP1/CEN-20 ratio. Notably, the correlation for the TOP1 $\mathrm{CN}$ was superior to the TOP1/CEN-20 ratio, which is consistent with the data in our study [18]. Our results demonstrated a borderline significant association 


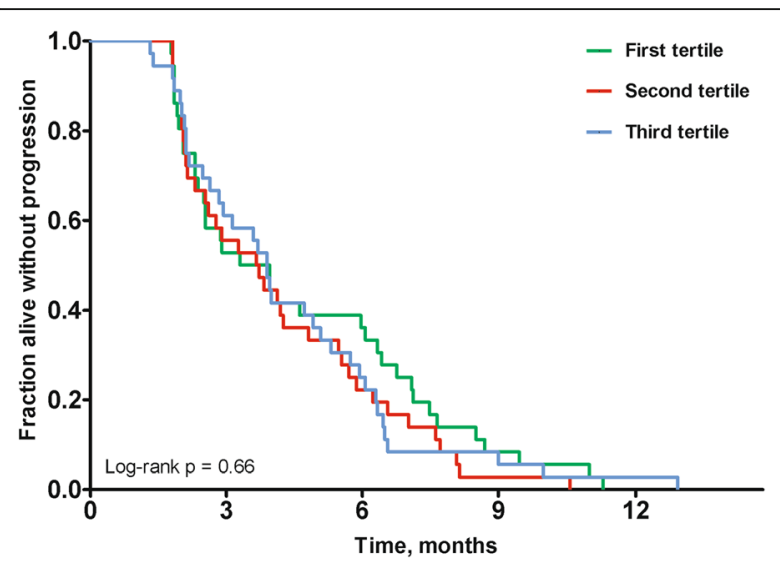

Fig. 4 Kaplan-Meier plot for progression-free survival as a function of TOP1 gene copy number. Patients were divided into tertiles by TOP1 copy number

between TOP1 $\mathrm{CN}$ and objective response $(p=0.08)$, which is in accordance with the results by Nygaard et al. $(p=0.07)$ [19]. However, this result was not further supported as there were no association between TOP1 CN and PFS. Regarding the ratios of TOP1/CEN-20 or TOP1/CEN-2 and response to irinotecan we found no associations to irinotecan response. However, the TOP1 $\mathrm{CNs}$ was significantly correlated to the CEN-20 CNs which may mask TOP1 amplification. Collectively, these findings could support the use of gene $\mathrm{CN}$ only instead of a gene/CEN ratio, which is further supported from the clinically implemented human epidermal receptor-2 (HER2) FISH analysis where only HER-2 CN can be reported without the ratio in cases with coamplification of HER-2 and CEN-17 [26].

The patients in this study constituted a selected group with a good prognosis as they were identified from a national cohort characterized by all patients having received third line treatment (irinotecan + cetuximab). Besides a long median OS (16.4 months) as could be expected in this selected group, the clinical data in this study corresponded to what have previously been reported from studies including irinotecan second-line monotherapy with RR of 10\% [27]. With 108 patients and RR of only $9 \%$ we have limited power to detect a predictive value of TOP1 as a biomarker for response. For that reason, we also investigated clinical benefit rate.

A major obstacle for obtaining a high level of evidence for predictive biomarkers is the lack of well-defined cutoff values for overexpression and gene amplification. Even for a well-established biomarker such as HER-2 in breast cancer, cut-off values are still discussed and have changed over time.

The level of Top1 protein expression could be useful as a predictive biomarker for the response to Top1 targeting chemotherapy. A number of studies have investigated this possibility in patients having $\mathrm{CRC}$, but with conflicting results $[10-14]$. These inconsistent results may partly be explained by the fact that immunohistochemistry (IHC) requires validated antibodies as well as standardized protocols, both of which have been difficult to obtain for Top1. Accordingly, Top1 IHC has not yet reached the required level for clinical implementation.

Another aspect is the different DNA repair mechanisms activated upon DNA damage which may in part explain why TOP1 CN alone could not predict response. A study reported that the expression of aprataxin, a histidine triad domain superfamily protein involved in DNA repair, in 30 CRC cell lines was correlated with sensitivity to irinotecan [28]. Another repair pathway, the tyrosyl-DNA-phosphodiesterase I (TDP1) was investigated in clinical CRC samples and cell lines. The authors reported that TDP1 is involved in the resolution of DNA damage associated with Top1 poisons and that the protein expression levels of TDP1 or Top 1 alone were not associated with sensitivity to irinotecan [29].

A robust correlation between TOP1 CN status, gene expression level, protein expression level and -activity in cancer cell lines $[9,30]$ and cancer tissues [31, 32] has been reported. Accordingly, the TOP1 CN may be a useful "proxy" predictive biomarker for Top1 targeting drugs. One concern in this study is whether the TOP1 $\mathrm{CN}$ determined in primary tumors correspond to the $\mathrm{CN}$ in the treated metastases. In our study primary tumors were analyzed in order to test our hypothesis. However, the majority of the treated tumor volumes in these patients consisted of metastases. Conflicting results have been reported regarding the concordance between primary tumor and metastases. Two studies reported a significantly higher Top1 expression in the metastases than in the corresponding primary tumor [33] [34]. Yet, other studies investigating TOP1 mRNA [35] and Top1 protein levels [36] have reported concordance between primary tumors and metastases. Another concern is the impact of prior treatments as it may change the biologic profile between the primary and recurrent tumors. An ideal design to overcome the difficulties mentioned above would be to assess TOP1 $\mathrm{CN}$ in the metastasis from $\mathrm{mCRC}$ prior to first-line irinotecan-containing therapy.

\section{Conclusions}

We verified a borderline significant association between increasing TOP1 $\mathrm{CN}$ and objective response to irinotecan monotherapy in $\mathrm{mCRC}$ patients as previously reported. Applying CEN-20 and CEN-2, to TOP1, did not provide further information to assist a biomarker driven patient stratification, suggesting that other biomarkers should be paired with TOP1 CN. 


\section{Acknowledgements}

The authors wish to thank Birgitte Christiansen and Hanne Michelsen for excellent assistance in data collecting. The bioanalysts at the Molecular Unit, Department of Pathology, Herlev Hospital for assistance in TMA production and FISH analyzes. Statistician Tobias Wirenfeldt Klausen, Department of Hematology, Herlev Hospital for assistance with statistical analysis. Sofie Seit Jespersen, Jakob Johansen and Frederik Schaltz-Buchholzer for assistance with the database. This work was supported by The Danish Cancer Research Foundation and the I.M. Daehnfeldt Foundation.

\section{Funding}

The Danish Cancer Research Foundation and I.M Daehnfeldt Foundation.

\section{Availability of data and materials}

The materials described in this article including raw data are deposited at the department of Oncology, Herlev University Hospital, Denmark and will be available to any scientist on request.

\section{Authors' contributions}

Conception and design: JAP, EH, DLN, BVJ, NB, TSP, DL, JS. Provision of study materials or patients: JAP, DL, EH, DLN, BVJ, TSP, MY, PP, LST. Collection and assembly of data: JAP, DL, DLN, EH, PP, LST, MY. Data analysis and interpretation: JAP, JS, NB, TSP, EH, DL, DLN. Manuscript writing and critical revision: JAP wrote first draft. DLN, JS, NB, EH and LST revised it critically. Final approval of manuscript: All authors.

\section{Competing interests}

The authors declare that they have no competing interests.

\section{Consent to publication}

All data reported in this article are entirely unidentifiable and thus no consent for publication has been obtained.

\section{Ethics approval and consent to participate}

The study was approved by the Research Ethics Committee of Copenhagen (H-KA-20060094)." In Denmark approval for retrospective studies can be obtained without having signed informed consent if 1) the data generated do not influence future treatments of the patients 2 ) if the study does not represent any harm to the patients. All the patients in this study are deceased.

\section{Author details}

'Department of Oncology, Herlev Hospital, University of Copenhagen, Herlev Ringvej 75, DK-2730 Herlev, Denmark. ${ }^{2}$ Department of Pathology, Herlev Hospital, University of Copenhagen, Herlev Ringvej 75, DK-2730 Herlev, Denmark. ${ }^{3}$ Department of Oncology, Odense University Hospital, Sdr. Boulevard 29, 5000 Odense C, Denmark. ${ }^{4}$ Faculty of Health and Medical Sciences, Department of Veterinary Disease Biology, Section for Molecular Disease Biology and Sino-Danish Breast Cancer Research Centre, University of Copenhagen, Copenhagen, Denmark. ${ }^{5}$ Department of Oncology, Aalborg University Hospital, Hobrovej 18-22, 9100 Aalborg, Denmark.

\section{Received: 27 January 2016 Accepted: 14 December 2016} Published online: 11 January 2017

\section{References}

1. Haggar FA, Boushey RP. Colorectal cancer epidemiology: incidence, mortality, survival, and risk factors. Clin Colon Rectal Surg. 2009;22(4):191-7.

2. Siegel R, Desantis C, Jemal A. Colorectal cancer statistics, 2014. CA Cancer J Clin. 2014;64(2):104-17.

3. Schmoll HJ, Van Cutsem E, Stein A, Valentini V, Glimelius B, Haustermans K, Nordlinger B, van de Velde CJ, Balmana J, Regula J, et al. ESMO Consensus Guidelines for management of patients with colon and rectal cancer. a personalized approach to clinical decision making. Ann Oncol. 2012;23(10): 2479-516.

4. Tournigand C, Andre T, Achille E, Lledo G, Flesh M, Mery-Mignard D, Quinaux E, Couteau C, Buyse M, Ganem G, et al. FOLFIRI followed by FOLFOX6 or the reverse sequence in advanced colorectal cancer: a randomized GERCOR study. J Clin Oncol. 2004;22(2):229-37.

5. Burstein HJ, Mangu PB, Somerfield MR, Schrag D, Samson D, Holt L, Zelman D, Ajani JA, American Society of Clinical O. American Society of Clinical
Oncology clinical practice guideline update on the use of chemotherapy sensitivity and resistance assays. J Clin Oncol. 2011;29(24):3328-30.

6. Pommier $Y$, Leo $E$, Zhang $H$, Marchand C. DNA topoisomerases and their poisoning by anticancer and antibacterial drugs. Chem Biol. 2010; 17(5):421-33.

7. Gilbert DC, Chalmers AJ, El-Khamisy SF. Topoisomerase I inhibition in colorectal cancer: biomarkers and therapeutic targets. Br J Cancer. 2012;106(1):18-24.

8. Ashour ME, Atteya R, El-Khamisy SF. Topoisomerase-mediated chromosomal break repair: an emerging player in many games. Nat Rev Cancer. 2015; 15(3):137-51.

9. McLeod HL, Keith WN. Variation in topoisomerase I gene copy number as a mechanism for intrinsic drug sensitivity. Br J Cancer. 1996;74(4):508-12.

10. Jansen WJ, Zwart B, Hulscher ST, Giaccone G, Pinedo HM, Boven E. CPT-11 in human colon-cancer cell lines and xenografts: characterization of cellular sensitivity determinants. Int J Psychol. 1997;70(3):335-40.

11. Braun MS, Richman SD, Quirke P, Daly C, Adlard JW, Elliott F, Barrett JH, Selby P, Meade AM, Stephens RJ, et al. Predictive biomarkers of chemotherapy efficacy in colorectal cancer: results from the UK MRC FOCUS trial. J Clin Oncol. 2008;26(16):2690-8.

12. Maughan TS, Meade AM, Adams RA, Richman SD, Butler R, Fisher D, Wilson RH, Jasani B, Taylor GR, Williams GT, et al. A feasibility study testing four hypotheses with phase II outcomes in advanced colorectal cancer (MRC FOCUS3): a model for randomised controlled trials in the era of personalised medicine? Br J Cancer. 2014;110(9):2178-86.

13. Koopman M, Knijn N, Richman S, Seymour M, Quirke $P$, van Tinteren $H$, van Krieken JHJM, Punt CJA, Nagtegaal ID. 6003 The correlation between Topoisomerase-I (Topo1) expression and outcome of treatment with capecitabine and irinotecan in advanced colorectal cancer (ACC) patients (pts) treated in the CAIRO study of the Dutch Colorectal Cancer Group (DCCG). Eur J Cancer Suppl. 2009;7(2):321-2.

14. Romer MU, Jensen NF, Nielsen SL, Muller S, Nielsen KV, Nielsen HJ, Brunner N. TOP1 gene copy numbers in colorectal cancer samples and cell lines and their association to in vitro drug sensitivity. Scand J Gastroenterol. 2012;47(1):68-79.

15. Fan B, Dachrut S, Coral H, Yuen ST, Chu KM, Law S, Zhang L, Ji J, Leung SY, Chen X. Integration of DNA copy number alterations and transcriptional expression analysis in human gastric cancer. PLoS One. 2012;7(4):e29824

16. Ryan D, Rafferty M, Hegarty S, O'Leary P, Faller W, Gremel G, Bergqvist M, Agnarsdottir M, Stromberg S, Kampf C, et al. Topoisomerase I amplification in melanoma is associated with more advanced tumours and poor prognosis. Pigment Cell Melanoma Res. 2010;23(4):542-53.

17. Tanner MM, Tirkkonen M, Kallioniemi A, Collins C, Stokke T, Karhu R, Kowbel D, Shadravan F, Hintz M, Kuo WL, et al. Increased copy number at 20q13 in breast cancer: defining the critical region and exclusion of candidate genes. Cancer Res. 1994:54(16):4257-60.

18. Romer MU, Nygard SB, Christensen IJ, Nielsen SL, Nielsen KV, Muller S, Smith $\mathrm{DH}$, Vainer B, Nielsen HJ, Brunner N. Topoisomerase 1(TOP1) gene copy number in stage III colorectal cancer patients and its relation to prognosis. Mol Oncol. 2013;7(1):101-11.

19. Nygard SB, Christensen IJ, Nielsen SL, Nielsen HJ, Brunner N, Spindler KL. Assessment of the topoisomerase I gene copy number as a predictive biomarker of objective response to irinotecan in metastatic colorectal cancer. Scand J Gastroenterol. 2013;49(1):84-91.

20. Tanner MM, Grenman S, Koul A, Johannsson O, Meltzer P, Pejovic T, Borg A, Isola JJ. Frequent amplification of chromosomal region 20q12-q13 in ovarian cancer. Clin Cancer Res. 2000;6(5):1833-9.

21. Smith DH, Christensen IJ, Jensen NF, Markussen B, Romer MU, Nygard SB, Muller S, Nielsen HJ, Brunner N, Nielsen KV. Mechanisms of topoisomerase I (TOP1) gene copy number increase in a stage III colorectal cancer patient cohort. PLoS One. 2013;8(4):e60613.

22. Therasse P, Arbuck SG, Eisenhauer EA, Wanders J, Kaplan RS, Rubinstein L, Verweij J, Van Glabbeke M, van Oosterom AT, Christian MC, et al. New guidelines to evaluate the response to treatment in solid tumors. European Organization for Research and Treatment of Cancer, National Cancer Institute of the United States, National Cancer Institute of Canada. J Natl Cancer Inst. 2000:92(3):205-16.

23. Altman DG, McShane LM, Sauerbrei W, Taube SE. Reporting recommendations for tumor marker prognostic studies (REMARK): explanation and elaboration. BMC Med. 2012;10:51. 
24. Beroukhim R, Mermel CH, Porter D, Wei G, Raychaudhuri S, Donovan J, Barretina J, Boehm JS, Dobson J, Urashima M, et al. The landscape of somatic copy-number alteration across human cancers. Nature. 2010; 463(7283):899-905.

25. Nygard SB, Christensen IJ, Nielsen SL, Nielsen HJ, Brunner N, Spindler KL. Assessment of the topoisomerase I gene copy number as a predictive biomarker of objective response to irinotecan in metastatic colorectal cancer. Scand J Gastroenterol. 2014:49(1):84-91.

26. Hanna WM, Ruschoff J, Bilous M, Coudry RA, Dowsett M, Osamura RY, Penault-Llorca F, van de Vijver M, Viale G. HER2 in situ hybridization in breast cancer: clinical implications of polysomy 17 and genetic heterogeneity. Mod Pathol. 2014;27(1):4-18.

27. Oostendorp LJ, Stalmeier PF, Pasker-de Jong PC, Van der Graaf WT, Ottevanger PB. Systematic review of benefits and risks of second-line irinotecan monotherapy for advanced colorectal cancer. Anteicancer Drugs. 2010;21(8):749-58.

28. Dopeso H, Mateo-Lozano S, Elez E, Landolfi S, Ramos Pascual FJ, HernandezLosa J, Mazzolini R, Rodrigues P, Bazzocco S, Carreras MJ, et al. Aprataxin tumor levels predict response of colorectal cancer patients to irinotecanbased treatment. Clin Cancer Res. 2010;16(8):2375-82.

29. Meisenberg C, Gilbert DC, Chalmers A, Haley V, Gollins S, Ward SE, El-Khamisy SF. Clinical and cellular roles for TDP1 and TOP1 in modulating colorectal cancer response to irinotecan. Mol Cancer Ther. 2015;14(2):575-85.

30. Reinhold WC, Mergny JL, Liu H, Ryan M, Pfister TD, Kinders R, Parchment R, Doroshow J, Weinstein JN, Pommier Y. Exon array analyses across the $\mathrm{NCl}-60$ reveal potential regulation of TOP1 by transcription pausing at guanosine quartets in the first intron. Cancer Res. 2010;70(6):2191-203.

31. Yu J, Miller R, Zhang W, Sharma M, Holtschlag V, Watson MA, McLeod HL. Copy-number analysis of topoisomerase and thymidylate synthase genes in frozen and FFPE DNAs of colorectal cancers. Pharmacogenomics. 2008;9(10): 1459-66.

32. Husain I, Mohler JL, Seigler HF, Besterman JM. Elevation of topoisomerase I messenger RNA, protein, and catalytic activity in human tumors: demonstration of tumor-type specificity and implications for cancer chemotherapy. Cancer Res. 1994;54(2):539-46.

33. Silvestris N, Simone G, Partipilo G, Scarpi E, Lorusso V, Brunetti AE, Maiello E, Paradiso A, Mangia A. CES2, ABCG2, TS and Topo-I primary and synchronous metastasis expression and clinical outcome in metastatic colorectal cancer patients treated with first-line FOLFIRI regimen. Int J Mol Sci. 2014;15(9):15767-77.

34. Tsavaris N, Lazaris A, Kosmas C, Gouveris P, Kavantzas N, Kopterides P, Papathomas T, Agrogiannis G, Zorzos H, Kyriakou V, et al. Topoisomerase I and llalpha protein expression in primary colorectal cancer and recurrences following 5-fluorouracil-based adjuvant chemotherapy. Cancer Chemother Pharmacol. 2009;64(2):391-8.

35. Vallbohmer D, labal S, Yang DY, Rhodes KE, Zhang W, Gordon M, Fazzone W, Schultheis AM, Sherrod AE, Danenberg KD, et al. Molecular determinants of irinotecan efficacy. Int J Cancer. 2006:119(10):2435-42.

36. Boonsong A, Curran S, McKay JA, Cassidy J, Murray GI, McLeod HL. Topoisomerase I protein expression in primary colorectal cancer and lymph node metastases. Hum Pathol. 2002;33(11):1114-9.

\section{Submit your next manuscript to BioMed Central and we will help you at every step:}

- We accept pre-submission inquiries

- Our selector tool helps you to find the most relevant journal

- We provide round the clock customer support

- Convenient online submission

- Thorough peer review

- Inclusion in PubMed and all major indexing services

- Maximum visibility for your research

Submit your manuscript at www.biomedcentral.com/submit
Biomed Central 